Artículo de investigación.

Cómo citar: D. Duarte, K. López, y S. Meneses,

"Modelo de probabilidad de accidente en bicicleta. Caso de estudio Engativá, Bogotá”, Inventum, vol. 14, no. 27, pp. 51-65. Disponible en doi:10.26620/uniminuto.inventum.14.27.2019.51-65

Editorial: Corporación Universitaria Minuto de Dios - UNIMINUTO.

ISSN: $1909-2520$

eISSN: $2590-8219$

Fecha de recibido: mayo 01 de 2019 Fecha de aprobado: junio 30 de 2019 Fecha de publicación: septiembre 01 de 2019

Conflicto de intereses: los autores han declarado que no existen intereses en competencia.

\section{Modelo de probabilidad de accidente en bicicleta. Caso de estudio Engativá, Bogotá}

\author{
Modelo de probabilidade de acidente \\ de bicicleta. Estudo de caso \\ Engativá, Bogotá
}

\author{
Probability model of bicycle accident. \\ Case study Engativá, Bogotá
}

\section{Resumen}

En Bogotá se ha incrementado el uso de la bicicleta y, con este, los índices en accidentalidad, ya que para el 2017 se presentó una cifra de 71 ciclistas muertos y 1.271 lesionados. A pesar de que existen campañas de seguridad vial, la cantidad de accidentes no disminuye. Con base en una revisión del estado del arte, en los últimos 5 años se observa que hay registros de la accidentalidad explicada de manera monocausal pero no un análisis integral de las diferentes causas en contexto.

Se presenta una investigación descriptiva que desarrolla un modelo que explica qué variables influyen en la ocurrencia de un accidente en bicicleta, para lo cual se utilizó información histórica de accidentalidad, facilitada por la Secretaría de Movilidad de Bogotá de los años 2015 a 2018, con las causales de accidentalidad reportadas. Se identificaron variables de accidentalidad de investigaciones del tema a nivel mundial, regional y local. Se compararon con información de encuestas a 280 biciusuarios de la localidad de Engativá.

Se obtiene un árbol de sucesos en que se asocian las variables y de cada una de ellas, el cálculo de la probabilidad frecuentista del suceso, cuyo cálculo está sujeto a la existencia o no de un accidente en bicicleta. Se aplicó el teorema de Bayes para explicar las variables condicionadas e integradas.

Deison Duarte

deisons.duartev@unilibrebog.edu.co Universidad Libre.

Katherine Lopez

katherinp.lopezr@unilibrebog.edu.co Universidad Libre

Sonia Meneses

sonial.menesesv@unilibre.edu.co

Universidad Libre

En conclusión, este modelo identifica las variables más significativas presentes en la accidentalidad en viaje en bicicleta, como base para orientar la formulación de campañas y políticas públicas más efectivas.

Palabras clave: bici-usuarios, movilidad, accidentalidad, árbol de sucesos, variables, bicicleta.

\section{Abstract}

In Bogotá, the use of the bicycle has increased and the rates of accidents have increased, with around 71 dead cyclists and 1271 injured by 2017 . 
Despite the existence of road safety campaigns, the accident figures are not consistent. Based on a review of the state of the art, in the last 5 years it is observed that there are records of the accident explained in a mono-causal way but not an integral analysis of the different causes in context.

This article presents a descriptive investigation that develops a model that explains which variables influence the occurrence of an accident on the bicycle trip, for which historical information was used of the accident of the Mobility Secretariat of Bogotá from 2015 to 2018, with the accident causes reported. Accident variables of investigations of the topic at the global, regional and local level were identified. They were compared with survey information to 520 bicycle user of the Town of Engativá.

An Event tree is applied, associating with it the variables and of each of them the calculation of the frequentistic probability of the event, whose calculation is subject to the existence or not of an accident on the bicycle trip. Bayes theorem was applied to explain the conditioned and integrated variables.

In conclusion, this model identifies the most significant variables present in the accident on a bicycle trip, as a basis to guide the formulation of more effective public campaigns and policies.

Keywords: Bike-users, mobility, accident, event tree, variables, bicycle.

\section{Resumo:}

Em Bogotá, o uso da bicicleta aumentou e, com ela, os índices de acidentes, já que em 2017 havia 71 ciclistas mortos e 1.271 feridos. Embora existam campanhas de segurança rodoviária, o número de acidentes não diminui. Com base em uma revisão do estado da arte, nos últimos 5 anos, observa-se que há registros do acidente explicados de forma monocausal, mas não uma análise integral das diferentes causas no contexto.

Este artigo apresenta uma investigação descritiva que desenvolve um modelo que explica quais variáveis influenciam a ocorrência de um acidente no passeio de bicicleta, para o qual foram utilizadas informações históricas do acidente, fornecidas pela Secretaria de Mobilidade de Bogotá de los anos de 2015 a 2018, com as causas do acidente relatadas. As variáveis de acidente foram identificadas para pesquisas sobre o tema em nível mundial, regional e local. Eles foram comparados com informações de pesquisas a 280 biciusarios da localidade de Engativá.

É aplicado para obter uma árvore de eventos alemães que associa as variáveis e a cada uma delas, o cálculo da probabilidade freqüencial do evento, cujo cálculo está sujeito à existência ou não de um acidente na viagem de bicicleta. O teorema de Bayes foi aplicado para explicar as variáveis condicionadas e integradas.

Concluindo, este modelo identifica as variáveis mais significativas presentes no acidente em um passeio de bicicleta, como base para orientar a formulação de campanhas e políticas públicas mais eficazes.

Palavras-chave: usuários de bicicleta, mobilidade, acidente, árvore de eventos, variáveis, bicicleta. 


\section{ANTECEDENTES}

En los últimos años, Colombia ha hecho énfasis en el uso de la bicicleta [1], debido a que ha tenido en cuenta sus múltiples beneficios [2]: mejoramiento de la salud de la población que las utiliza, es un medio de transporte sustentable [3] y sostenible, no crea congestión en las vías por su tamaño. Bogotá cuenta con $500 \mathrm{~km}$ de ciclorrutas [5] como parte del POT, es la segunda ciudad con más kilómetros de infraestructura para uso de bicicletas seguido de Rosario, Argentina [6]. Pero tener esta cantidad de kilómetros de ciclorrutas en la ciudad no es suficiente para disminuir el porcentaje de lesionados y fatalidades, como se muestra a continuación.

Figura 1. Porcentaje de muertes y lesionados según medio de transporte

\section{MUERTES}

Ciclistas 2017 - Objeto de choque

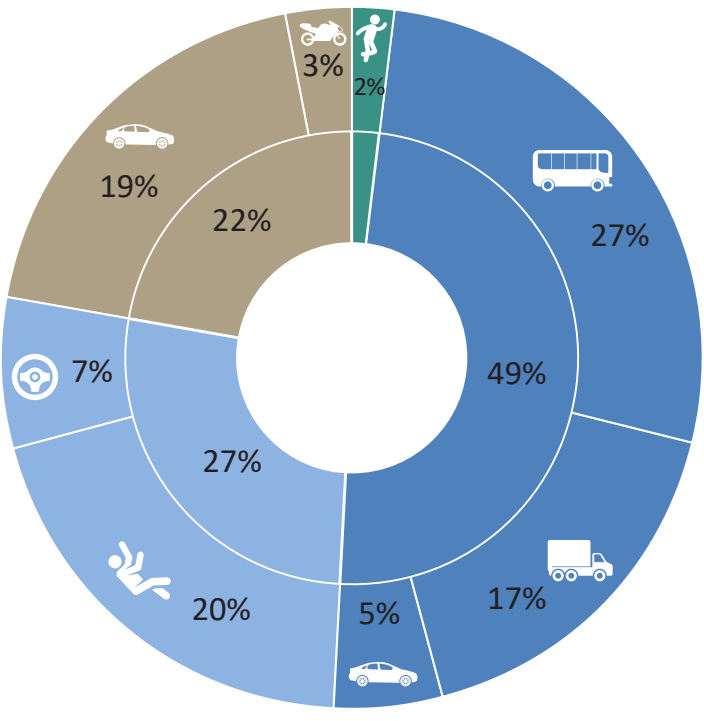

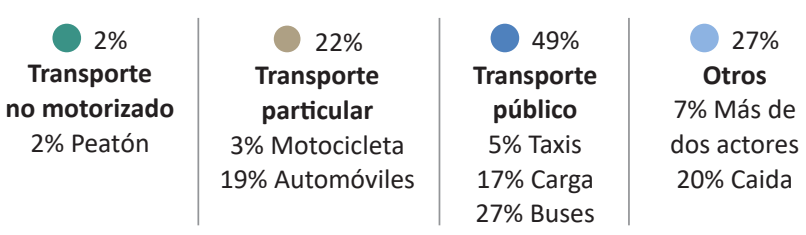

LESIONADOS

Ciclistas 2017 - Objeto de choque
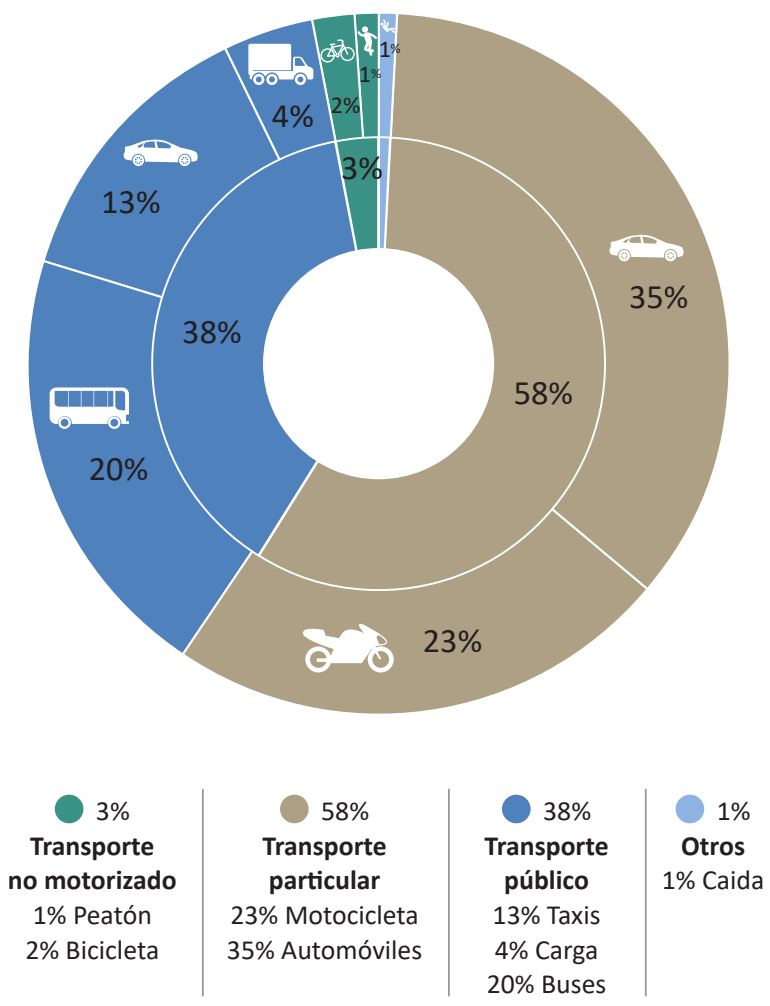

Fuente: [10]

Según el Instituto Nacional de Medicina Legal y Ciencias Forenses, el 5 \% representa los lesionados y el $7 \%$ los biciusuarios muertos [7]; con el transcurso de los años se evidencia un crecimiento en el uso de las bicicletas y al mismo tiempo la tasa de accidentes [8]. En la localidad de Engativá se encuentra la población biciusuaria con mayor número de viajes por día en bicicleta, según el reporte de la Secretaría Distrital de Movilidad [8] y [9], pero así mismo esta es la segunda localidad con mayor número de siniestros [10], en el periodo de 2016 a 2018 ocurrieron 680 siniestros en bicicleta en este sector de Bogotá, según el reporte obtenido de la Secretaría Distrital de Movilidad [11].

El Gobierno se enfocó en impulsar este medio de transporte brindando más infraestructura, pero no tomó en cuenta que hay diferentes factores que están ocasionando que aumente la probabilidad de que los biciusuarios sufran un accidente porque no hay política pública en prevención de accidentes, tan solo publicaciones de cómo ser un buen biciusuario [12]. Con base en esta problemática, en este artículo se muestran los resultados de la 
investigación, donde inicialmente se empleó la información secundaria para establecer las variables que influyen en la accidentalidad durante el proceso de movilidad de biciusuarios, se hallaron diferentes impactos en la accidentalidad, pero cada estudio tiene un enfoque diferente como inclusión, infraestructura o comportamiento, o estudios con una variable de accidentalidad o monocausales, lo cual no es coherente con la descripción de los eventos de accidentes de los biciusuarios encuestados.

Por ello se recurrió a la información de las encuestas realizadas por la Secretaría Distrital de
Movilidad y se procedió a efectuar otra encuesta a la población de la localidad de Engativá, tomando una muestra representativa para comparar los resultados obtenidos.

El objetivo de este proyecto consiste en desarrollar un modelo que explique las variables de la accidentalidad en el proceso de movilidad en bicicleta en Engativá empleando el árbol de sucesos para poder hallar la probabilidad de que un accidente se materialice, dándole un enfoque multicausal teniendo en cuenta la realidad misma de ésta actividad.

Figura 2. Metodología de la investigación

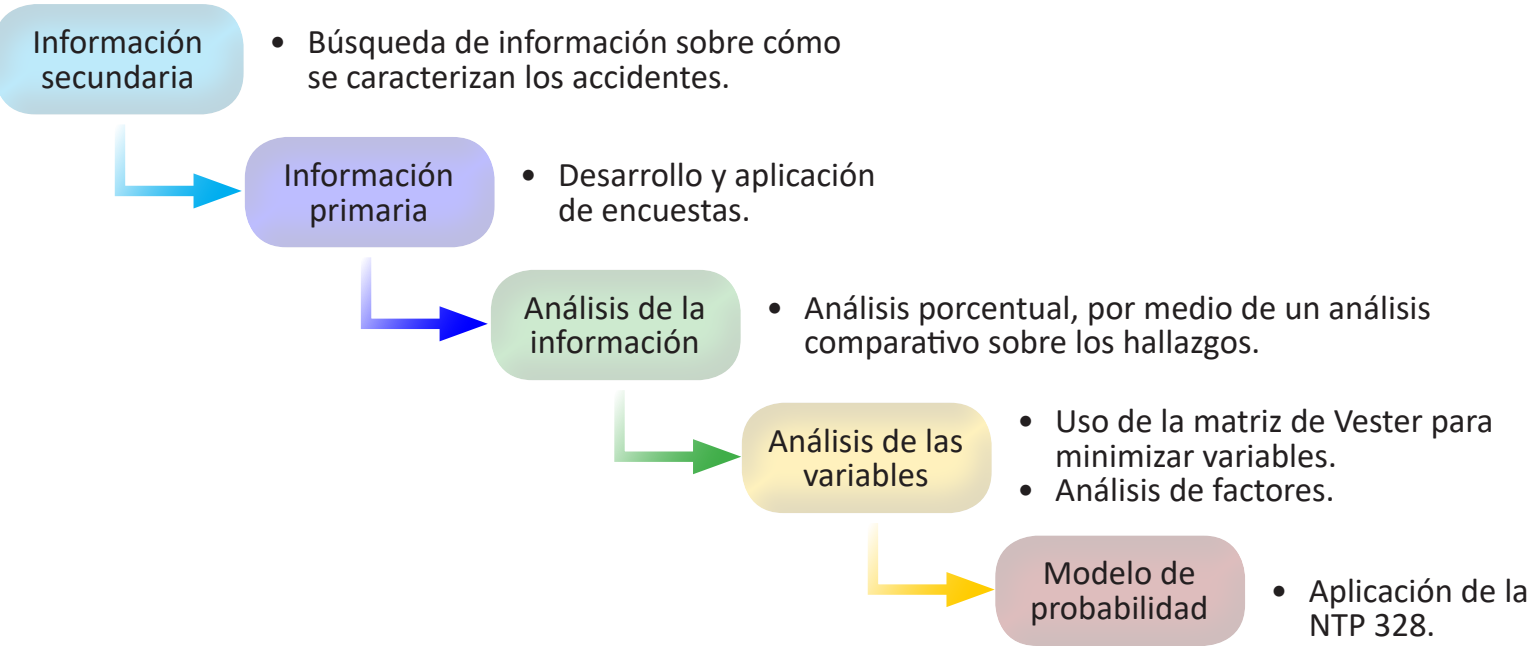

Fuente: elaboración propia.

\section{METODOLOGÍA}

La metodología empleada en esta investigación se presenta en las siguientes etapas las cual son resumidas en la figura 2 .

\section{A. Búsqueda de información secundaria}

Para llegar a la caracterización de las variables que afectan la accidentalidad de la movilidad en bicicleta, se hizo la búsqueda de la información secundaria en bases de datos sobre cómo se caracterizaban los accidentes. Estas variables desarrollaron un estado del arte con diferentes estudios a nivel nacional e internacional usando como referencia artículos científicos, revistas y reportes de movilidad para su posterior clasificación, los cuales sumaron 100 documentos.
Los principales documentos de donde se obtuvieron más datos fueron los siguientes con sus respectivas variables:

1. "Plan bici": infraestructura, exceso de velocidad, acción de terceros, transitar en contravía, girar bruscamente, embriaguez o drogas, accidente según género [3].

2. "How infrastructures can promote cycling in mediterranean cities: lessons from Sevilla" [13]: red vial ciclo rutas, intersecciones y calles sin ciclo vías, alta velocidad y falta de normas de seguridad.

3. “Incompetent or too competent? Negotiating everyday cycling identities in a motor dominated society" [14]: imprudencia, ignorar señalización y viajar sin luces. 
4. Estudio sobre las estrategias de promoción de la bicicleta como medio de transporte en las ciudades españolas [15]: falta de ciclo ruta y timidez.

5. Infraestructures de transports et conflits [16]: mala distribución de espacios en la carretera, invasión de aceras para peatones, imprudencia de terceros y falta de educación para biciusuarios.

6. La bicicleta como medio de transporte puntos de vista de las personas usuarias y expertas [17]: inseguridad, imprudencia, lluvia, falta de señalización, mala infraestructura en las vías, acción de terceros, exceso de velocidad y falta de implementos de seguridad.

La Secretaría Distrital de Movilidad brindó una base de datos para la investigación, los cuales fueron de gran importancia ya que arrojaron antecedentes específicos de la localidad de Engativá en donde se realiza dicho estudio.

Después de obtener las variables influyentes, se procede a realizar la clasificación según su condición; prudencia, ciclista, bicicleta y entorno.

\section{B. Información primaria}

Para obtener la información primaria, se desarrollaron dos encuestas diferentes y se halló la población necesaria para aplicarlas mediante un el método probabilístico aleatorio simple, el cual arrojó un total de 218 biciusuarios a partir de la fórmula 1 y se logró aplicar a un total de 280 biciusuarios.

$$
n=\frac{z^{2} P Q N}{e^{2}(N-1)+z^{2} P Q}
$$

Donde:

$Z=$ nivel de confianza

$\mathrm{P}=$ proporción de accidentalidad

Q= proporción de no accidentalidad

$\mathrm{N}=$ población de biciusuarios $=500$ biciusuarios

$\mathrm{E}=$ nivel de error muestral

$$
n=\frac{1,96^{2} * 0,5 * 0,5 * 500}{0,05^{2}(500-1)+1,96^{2} * 0,5 * 0,5}
$$

\section{$n=218$ Biciusuarios}

Debido a que el $50 \%$ de los encuestados alguna vez se ha accidentado, se tomó la proporción de accidentalidad y de no accidentalidad como un 0,5; la población de biciusuarios se obtuvo según la base de datos de los biciusuarios registrados en la Universidad Libre hasta el momento de la realización de la primera encuesta. Se tomó un nivel de error bajo de un 0,05 para dar más certeza a los resultados, y un nivel de confiabilidad del $97,5 \%$ que, al momento de calcular la $z$, es decir la distribución normal da 1,96; dando así un total de 218 encuestas que se deben realizar para tener una buena muestra; y se realizaron para más confiabilidad 280.

El estudio se desarrolló, primero con información secundaria y luego la información primaria para ratificar lo anterior. Para obtener dicha información se usaron métodos estadísticos para la construcción de las encuestas y para conocer el tamaño de la muestra. Teniendo las variables arrojadas de la información primaria y secundaria, se procedió a analizarlas por medio de un diagrama. Se realizó un análisis porcentual por medio de un análisis comparativo de los hallazgos, y se conocieron de esta manera las variables que más influencia tienen en la accidentalidad. 
Figura 3. Causas probables de un accidente según informe de movilidad

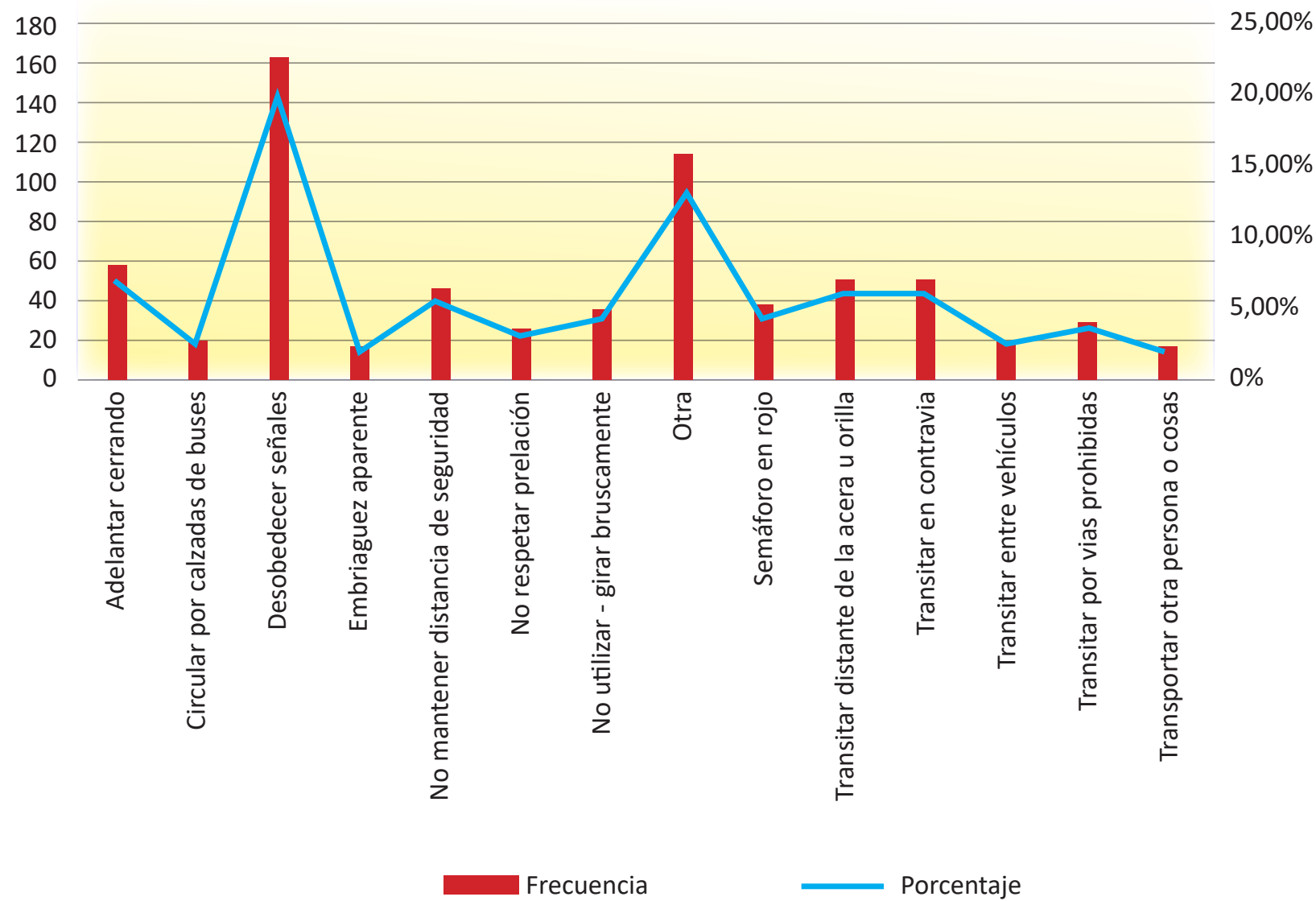

Fuente: Secretaría Distrital de Movilidad. Tomado de los datos de la oficina de información sectorial SDM, 2013, del documento técnico de soporte de la Secretaría Distrital de Movilidad.

Debido a que se obtuvieron más de 15 variables que influenciaban la accidentalidad, se optó por aplicar la matriz de Vester para priorizar las variables críticas. Teniendo las variables con mayor influencia, se procedió a aplicar la NTP 328 de análisis de riesgos mediante el árbol de sucesos [18], el cual desarrolla un diagrama secuencial a partir de sucesos iniciadores no deseados para conocer lo que puede acontecer conociendo la probabilidad en este caso del accidente.

\section{RESULTADOS Y DISCUSIÓN}

Información secundaria: teniendo las variables que afectan la accidentalidad, anteriormente nombradas, se clasificaron de la siguiente manera: ciclista, infraestructura, entorno e imprudencia. Según el estudio realizado por la secretaria de movilidad del 2016 [19].
Las variables proporcionalmente relevantes son: desobedecer señales (el cual comprende cuando hacen caso omiso a las señales de tránsito); adelantar cerrando (cuando se está adelantando un carro moto o bicicleta sin dejar una distancia prudente entre el otro vehículo y la bicicleta), y transitar en contravía, tal como se evidencia en la figura 3 con sus respectivas frecuencias y porcentajes de participación.

\section{A. Unificación de las variables}

Por medio de un criterio según la caracterización de riesgos de Jiménez Castro y Diaz Anacona [7], las variables más significativas fueron tomadas para su clasificación y análisis, a partir del porcentaje de ocurrencia, tabla 1. 
Las variables resaltadas en azul son las variables que se tienen en común en los dos tipos de información, y las verdes son variables que diferían en estos análisis, pero de igual manera se tuvieron en cuenta en el estudio por su porcentaje de ocurrencia; exceso de velocidad solo se encontró en la información primaria, estas diferencias son importantes para este estudio, para conocer realmente las variables influyentes.

Tabla 1. Variables significativas para un accidente

\begin{tabular}{c|l|l|c}
\multicolumn{1}{c|}{$\%$} & Variables según informe de secretaria de movilidad & Variables obtenidas según encuestas & $\%$ \\
\hline $5,61 \%$ & No mantener distancia de seguridad & No mantener distancia de seguridad & $12 \%$ \\
\hline $4,65 \%$ & Semáforo en rojo & Semáforo en rojo & $6 \%$ \\
\hline $19,57 \%$ & Desobedecer señales & Desobedecer señales & $6 \%$ \\
\hline $6,21 \%$ & Transitar en contravia & Transitar en contravia & $5 \%$ \\
\hline $7,04 \%$ & Adelantar cerrando & Adelantar cerrando & $4 \%$ \\
\hline $3,22 \%$ & No respetar prelación & No respetar prelación & $9 \%$ \\
\hline $13,72 \%$ & Otra & Exceso de velocidad & $19 \%$ \\
\hline $6,09 \%$ & Transitar distante de la acera u orilla de la calzada & Acción de automóviles o motos & $13 \%$ \\
\hline $4,30 \%$ & Girar bruscamente & Invasión de vías (personas, animales u objetos) & $7 \%$ \\
\hline
\end{tabular}

Fuente: Elaboración propia.

\section{B. Análisis de las variables}

Se optó por aplicar un análisis de factores para poder reducirlas: el número de variables según su índice de ocurrencia en la accidentalidad, para esto se aplica la siguiente fórmula:

$$
\% \text { factores }=\frac{\text { ocurrencia }}{\text { Eocurrencia de factores }}
$$

Esta se aplicó a cada una de las variables extraídas de los informes de la Secretaría Distrital de Movilidad, y se obtuvieron los resultados de la tabla 2 .

De este análisis se concluye que no mantener distancia de seguridad, desobedecer señales, transitar en contravía, adelantar cerrando y transitar distante de la acera u orilla de la calzada son las causales más primordiales a tener en cuenta según el criterio de tomar aquellos factores superiores a 5,5\%.

Tabla 2. Análisis de factores según ocurrencia

\begin{tabular}{c|l|c|c} 
& \multicolumn{1}{|c|}{ Causa } & Frecuencia & $\%$ \\
\cline { 2 - 4 } & No mantener distancia de seguridad & 47 & $5,61 \%$ \\
\hline P2 & No respetar el semáforo en rojo & 39 & $4,65 \%$ \\
\hline P3 & Desobedecer señales & 164 & $19,57 \%$ \\
\hline P4 & Transitar en contravía & 52 & $6,21 \%$ \\
\hline P5 & Adelantar cerrando & 59 & $7,04 \%$ \\
\hline P6 & No respetar prelación & 27 & $3,22 \%$ \\
\hline P7 & Exceso de velocidad & 12 & $1,43 \%$ \\
\hline P8 & Acción de automóviles o motos & 16 & $1,91 \%$ \\
\hline P9 & Invasión de vías (personas, animales u objetos) & 11 & $1,31 \%$ \\
\hline P10 & Transitar distante de la acera u orilla de la calzada & 51 & $6,09 \%$ \\
\hline P11 & Girar bruscamente & 36 & $4,30 \%$ \\
\hline P12 & Género & 3 & $0,36 \%$ \\
\hline & & & \\
\hline
\end{tabular}

Fuente: Elaboración propia. 
Debido a que aún se contaba con muchas variables, estas se priorizaron por medio de la matriz de Vester. Todos los datos fueron ingresados en esta matriz y se generó un plano con cuatro cuadrantes, en los cuales se clasificaron las variables en causas, efectos, problema central que son los críticos y los indiferentes. Las variables ubicadas en el cuadrante crítico (derecho superior) fueron las variables resultantes de esta herramienta: no mantener distancia de seguridad, desobedecer señales, adelantar cerrando, exceso de velocidad, imperfección en las vías. Para ratificar que los resultados no tuvieran error se realizó un análisis de consistencia.

Figura 4. Plano cartesiano de los resultados de la matriz de Vester

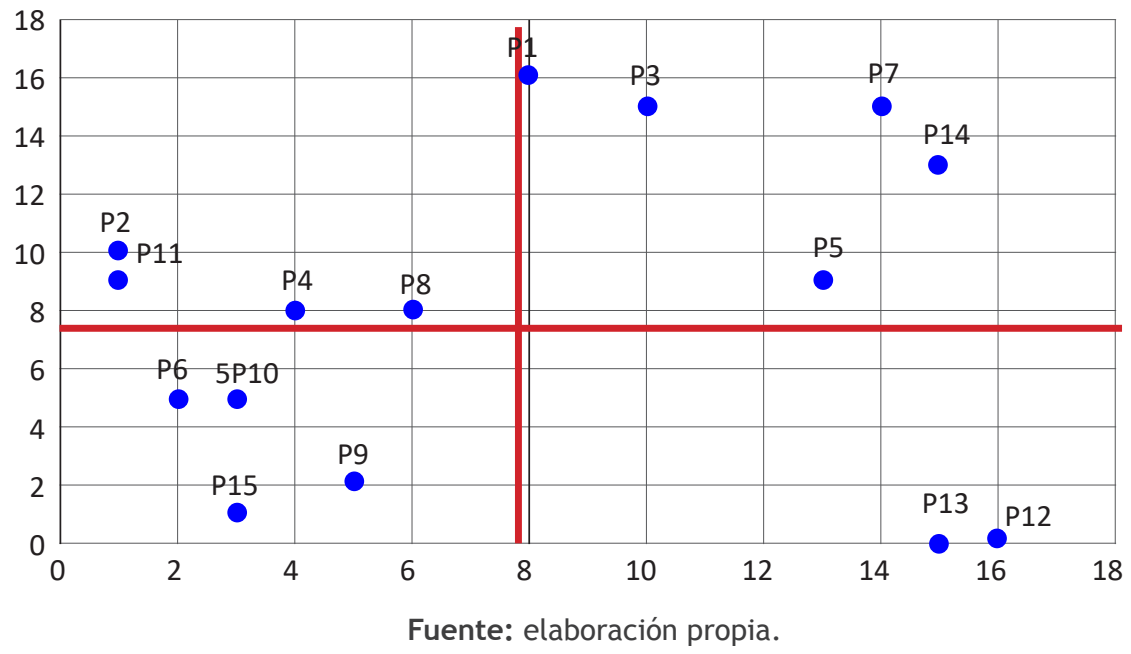

Algunas de estas variables tienen subvariables para poder desencadenar un accidente, estos eventos y subvariables están relacionadas y representados en el diagrama de la figura 5.

Figura 5. Variables más influyentes en la accidentalidad de biciusuarios en el proceso de movilidad en bicicleta

Sub-variable Variable Probabilidad

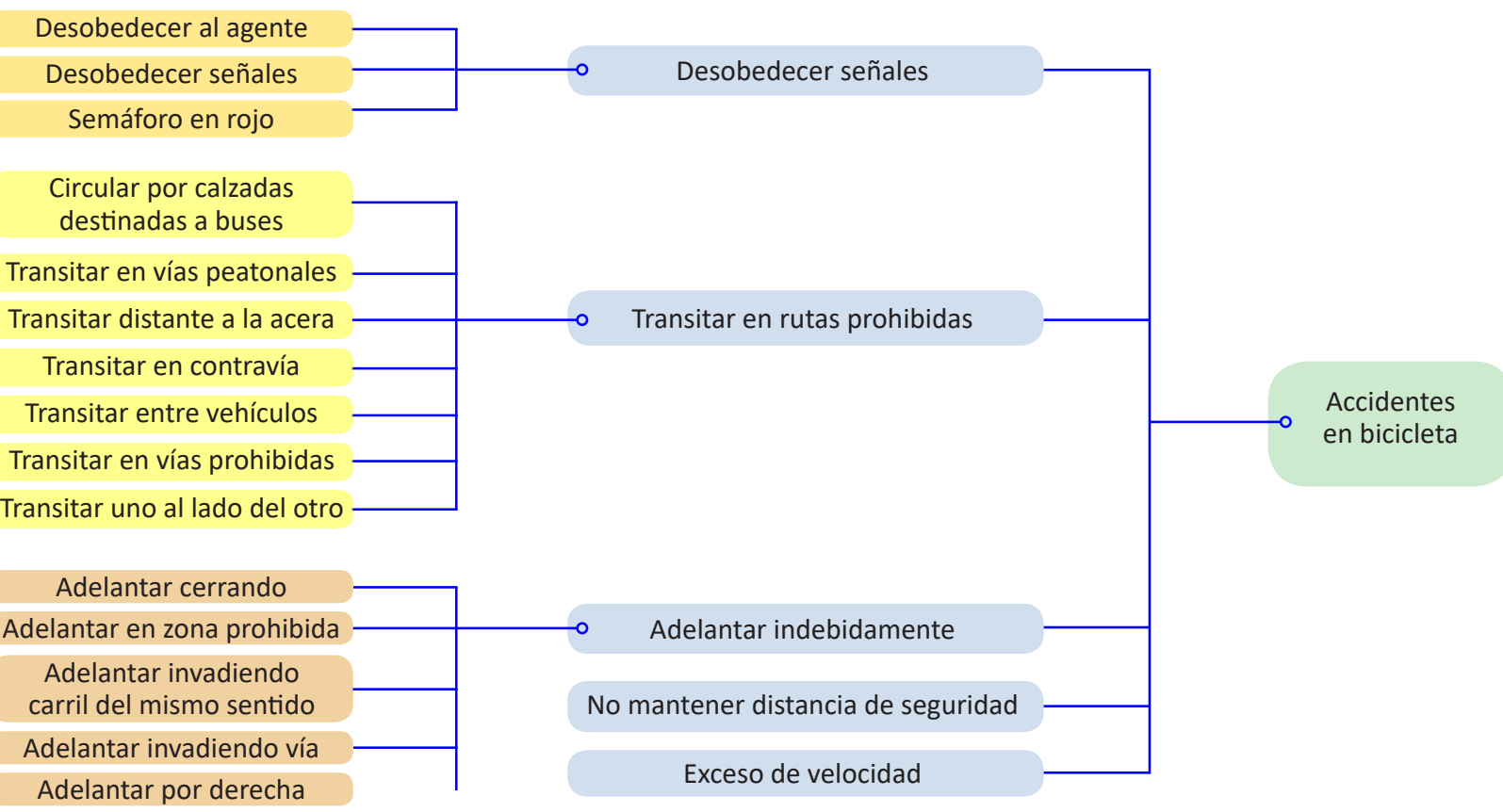

Fuente: elaboración propia. 
Para poder hallar las probabilidades de accidentarse, según las variables priorizadas anteriormente, se realizó un análisis de riesgos mediante el árbol de sucesos, teniendo en cuenta la NTP 328 [18].

A las variables priorizadas se les asignó una letra según el grado de incidencia para abreviar las relaciones de la siguiente manera:

\section{A Accidente}

\section{B Desobedecer señales}

C Transitar en contravía

\section{Adelantar cerrando}

E No mantener distancia de seguridad

F Exceso de velocidad

Hasta este punto se tuvieron frecuencias de ocurrencias de las variables de la accidentalidad. Se aplicó el concepto de probabilidad frecuencial. La cual hace referencia a la definición de probabilidad entendida como el cociente entre el número de casos favorables (accidentes ocurridos por esa variable) y el número de casos posibles, cuando el número de casos tiende a infinito. Matemáticamente la probabilidad frecuencial se expresa como:

\section{$\lim _{N \rightarrow \infty} \frac{S}{N}=P(s)$}

Donde:

s: es un suceso determinado

$\mathrm{N}$ : número total de sucesos

$\mathrm{P}(\mathrm{s})$ : es la probabilidad del suceso $\mathrm{s}$.

Intuitivamente esto se lee como el límite de la frecuencia cuando $\mathrm{n}$ tiende a infinito. En nuestro caso el número total de casos posibles es un número muy grande debido a la posibilidad de accidentarse que transcurre cada instante del viaje por cada viaje posible por cada ciclista y por cada ruta, lo cual representa un número muy grande.

Usando esta definición técnica, se aplicó a las variables identificadas y sus frecuencias, y se obtuvo una probabilidad frecuentista de accidentes registrados en Engativá por la Secretaría Distrital de Movilidad, la cual se halló dividiendo la frecuencia sobre el total de accidentes registrados, como se muestra en la tabla 3.

Tabla 3. Probabilidad de ocurrencia de cada variable

\begin{tabular}{|l|c|c|c|c|}
\hline \multicolumn{1}{|c|}{} & $\begin{array}{c}\text { TOTAL } \\
\text { ACCIDENTES }\end{array}$ & 838 & 838 & 533 \\
\hline \multicolumn{1}{|c|}{ ITEM } & REF & Frecuencia & $\%$ & $\%$ \\
\hline $\begin{array}{l}\text { Accidente en el proceso } \\
\text { de movilidad en bicicleta }\end{array}$ & $\mathrm{A}$ & 533 & $63,60 \%$ & $63,60 \%$ \\
\hline $\begin{array}{l}\text { Desobedecer señales } \\
\text { Transitar en contravía }\end{array}$ & $\mathrm{B}$ & 305 & $36,40 \%$ & $57,22 \%$ \\
\hline Adelantar cerrando & $\mathrm{C}$ & 80 & $9,55 \%$ & $15,01 \%$ \\
\hline $\begin{array}{l}\text { No mantener distancia de } \\
\text { seguridad }\end{array}$ & $\mathrm{E}$ & 89 & $10,62 \%$ & $16,70 \%$ \\
\hline Exceso de velocidad & $\mathrm{F}$ & 12 & $1,43 \%$ & $2,25 \%$ \\
\hline
\end{tabular}

Fuente: Elaboración propia.

Con base en la evaluación de dependencia de la de la matriz de Vester, se identificaron cuales variables eran independientes de las demás: B, C, D, E, $F$, y $G$. Además se identificaron aquellas variables que tenían una mayor dependencia con otras como la variable $\mathrm{F}$ y $\mathrm{C}$ que dependen de $\mathrm{B}$ o la Variable $\mathrm{E}$ y $F$ que dependen de la variable $D$.

Al ingresar al modelo también se tuvo que nombrar las variables que no afectan al evento en cada 
situación, por lo cual se nombra con una ( '), para poder diferenciarlo, es decir la no ocurrencia del evento.

La probabilidad de ocurrencia se halla con la siguiente fórmula:
1 - Frecuencia de la variable

Número total de accidentes

La aplicación de esta fórmula está en la tabla 4.

Tabla 4.Probabilidad de ocurrencia de cada variable

\begin{tabular}{|c|c|c|c|}
\hline REF & Frecuencia & $\%$ & $\%$ \\
\hline$\therefore$ 'B & 328 & $75,54 \%$ & $61,54 \%$ \\
\hline 'C & 353 & $78,52 \%$ & $66,23 \%$ \\
\hline 'D & 444 & $89,38 \%$ & $83,30 \%$ \\
\hline 'E & 486 & $94,39 \%$ & $91,18 \%$ \\
\hline 'F & 521 & $98,57 \%$ & $97,75 \%$ \\
\hline
\end{tabular}

Fuente: elaboración propia.

Se sumó a la importancia de la variables, la dinámica del proceso de accidentalidad, con base en las encuestas realizadas se concluyó que el accidente es la consecuencia de una serie de eventos, que representaremos en el modelo con las variables identificadas aquí con letras, que generan secuencias mutuamente excluyentes que se pueden representar con el árbol de sucesos.

Esta herramienta se utilizó para modelar, clasificar y calcular diversos escenarios del accidente posterior al evento desencadenante. Esta conclusión se basó en la búsqueda de modelos de ingeniería de confiabilidad, donde hay estudios que muestran cómo el árbol de sucesos se usa para analizar la dinámica de los eventos y su variabilidad [20] en términos probabilísticos. Se ha utilizado para análisis de eventos marinos [21], análisis de fallas en el sector petrolero [22], ente otros.

El árbol de sucesos, además, muestra gráficamente la relación entre cada variable (evento en la metodología) y su relación causa-efecto. La figura 6 muestra el resultado final de esta integración.

Dado que un accidente se podría ocasionar por la ocurrencia de una sola de las variables y excluir las demás, también se presentan evento fruto de la combinación de más de una variable. Lo cual debe estar evidenciado en el árbol de sucesos.

Para expresar esta dinámica se usa la teoría de conjuntos, que permite estudiar relaciones y propiedades entre diferentes colecciones de objetos al compararlas entre sí de diversas maneras [23]. Un conjunto es una colección de elementos, que es considerada en sí misma como un objeto. Para este estudio las variables se asumieron como conjuntos con letras mayúsculas (A, B, C, . . , Z). Ya que las variables representan una serie de elementos tales como: la variable B es desobeder señales, lo cual incluye en sí misma una serie de elementos tales como no conocer las señales, no verlas, no comprender, entre otras. 
Figura 6. Árbol de sucesos

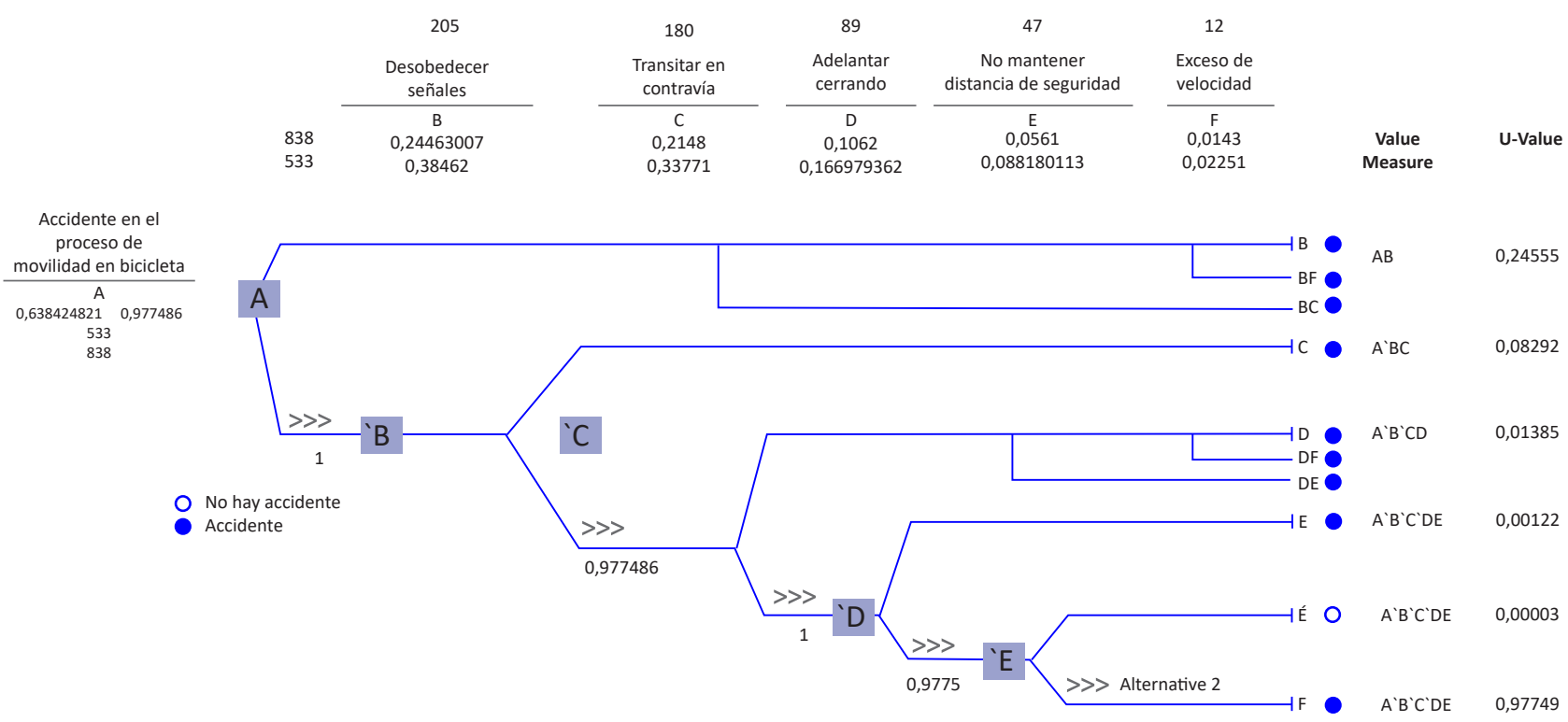

Fuente: elaboración propia.

Se usó en este análisis las propiedades de intersección entre conjuntos. Dichas propiedades son: Si A, $B$ y $C$ son conjuntos cualquiera, $U$ es el conjunto universal y $\emptyset$ el vacío, se tiene que:

1. $A \cap A=A$, la intercesión de un conjunto consigo mismo es el mismo conjunto.

2. $A \cap \emptyset=\emptyset$, al interceptar cualquier conjunto con el vacío se obtiene el vacío.

3. $A \cap U=A$, al interceptar al universal con cualquier conjunto se obtiene el conjunto.

4. $A \cap B=B \cap A$, la intersección entre conjuntos es conmutativa.

5. $(A \cap B) \cap C=A \cap(B \cap C)$, la intersección entre conjuntos es asociativa.

6. $A \cap B \subset A, A \cap B \subset B$, la intersección de $A$ y $B$ está incluido en $A$ y está incluido en $B$.

7. $A \subset B \longrightarrow(A \cup B) \subset A$, la intersección de un conjunto $A$ con un conjunto $B$ que lo contenga, deja a A inalterado.

8. $A \cap(B \cap C)=(A \cap B) \cup(A \cap C)$, la intersección de conjuntos distribuye con la unión de conjuntos.

9. $A \cup(B \cap C)=(A \cup B) \cap(A \cup C)$, la unión de conjuntos distribuye con la intersección de conjuntos.

Se aplicó al arbol de sucesos la ocurrencia de cada variable como sucesos mutuamente independientes y mutuamente excluyentes. Posteriormente, se aplicaron las diferentes relaciones de conjunto, para conocer cómo es el comportamiento de cada variable frente a otras, según el teorema de conjuntos.

Se calculó la probabilidad frecuencial de cada variable, y posteriormente de cada secuencia de variables en relación directa con el accidente de cada uno de los sucesos desencadenantes; como se presenta a continuación:

$A \cap B=0,24463 / 0,6384248=0,156178$

$A \cap B \cap F=0,24463^{*} 0,6384248^{*} 0,01=0,002236$

$\mathrm{A} \cap \mathrm{B} \cap \mathrm{C}=0,24463^{*} 0,6384248^{*} 0,21=0,033547$

$A \cap^{\prime} B \cap C=0,6384248^{*} 0,7553699^{*} 0,21=0,103585$

$A \cap{ }^{\prime} B \cap ' \cap D=0,6384248^{*} 0,7553699^{*} 0,7852029$

${ }^{*} 0,11=0,040216$

$A \cap$ ' $B \cap$ ' $C \cap D \cap E=0,6384248 * 0,7553699 * 0,7852029$

${ }^{*} 0,11{ }^{*} 0,06=0,002256$

$A \cap{ }^{\prime} B \cap ' C \cap D \cap F=0,6384248^{*} 0,7553699$

${ }^{*} 0,7852029 * 0,11{ }^{*} 0,01=0,000576$

$A \cap^{\prime} B \cap^{\prime} C \cap^{\prime} D \cap E=0,6384248 * 0,7553699$

${ }^{*} 0,785202{ }^{*} 0,8937947^{*} 0,06=0,018982$

$A \cap^{\prime} B \cap^{\prime} C C^{\prime} D \cap \prime E \cap F=0,6384248$ * 0,7553699

* $0,7852029 * 0,8937947 * 0,9439141$

* $0,01=0,004575$ 


\section{Validación del árbol de sucesos}

Las probabilidades condicionadas del árbol de sucesos se calcularon mediante el uso del teorema de Bayes, asociado a intersecciones de conjuntos, y que se define que para dos sucesos $A$ y $B$ donde $P(B)>0$, se definió la probabilidad condicionada de $A$ dado $B$ como $P(A \mid B)=P(A \cap B) / P(B)$. Se interpretó la probabilidad, como la probabilidad de $A$ suponiendo que B haya ocurrido.
Producto de este teorema son las probabilidades antes presentadas. Se continuó con la validación, tomando la frecuencia que tiene cada una de las variables con base en los datos históricos de accidentalidad de la Secretaría Distrital de Movilidad frente a las probabilidades obtenidas en el modelo. Para la validación del modelo se tomó la probabilidad frecuencial de cada una de las variables analizadas y correlacionadas en el árbol de sucesos con el fin de verificar que los resultados sean coherentes.

En la tabla 5 se presentan las comparaciones.

Tabla 5. Validación árbol de sucesos

\begin{tabular}{|c|c|c|c|c|c|c|}
\hline \multicolumn{4}{|c|}{ PROBABILIDADES } & & \multirow{3}{*}{$\begin{array}{l}\text { Diferencia } \\
\text { Secretaría }\end{array}$} & \multirow{3}{*}{$\begin{array}{l}\text { Diferencia árbol de } \\
\text { probabilidades }\end{array}$} \\
\hline \multicolumn{2}{|c|}{$\begin{array}{c}\text { Secretaría } \\
\text { (monocausal) }\end{array}$} & \multicolumn{2}{|c|}{$\begin{array}{l}\text { Árbol de probabilidades } \\
\text { (multicausal y condicionado) }\end{array}$} & & & \\
\hline$\%$ & \# & $\%$ & \# & & & \\
\hline 38,46 & 320 & $15,56 \%$ & 130 & $A \cap B$ & $36,01 \%$ & $58 \%$ \\
\hline 33,77 & 281 & $10,32 \%$ & 86 & $A \cap ' B \cap C$ & $36,01 \%$ & $109 \%$ \\
\hline 16,70 & 139 & $4,01 \%$ & 33 & $A \cap ' B \cap ' C \cap D$ & $36,01 \%$ & $167 \%$ \\
\hline 8,82 & 73 & $1,89 \%$ & 16 & $A \cap ' B \cap ' C \cap ' D \cap E$ & $36,01 \%$ & $198 \%$ \\
\hline 2,25 & 19 & $0,46 \%$ & 4 & $A \cap ' B \cap C \cap \cap D \cap ' E \cap F$ & $36.01 \%$ & $216 \%$ \\
\hline
\end{tabular}

Fuente: Elaboración propia.

Para este ejercicio se toman los 833 accidentes ocurridos desde el 2015 hasta junio del 2019, en donde está involucrada una bicicleta, para de esta manera, al observar el producto entre la probabilidad obtenida por la teoría de conjuntos de una causal y la cantidad total de accidentes se obtendría el número de accidentes que se ocasionarían dado que ocurren ciertos eventos. Por lo que se obtiene la gráfica que se muestra en la figura 8 , en la que se pueden apreciar los valores.

Figura 7 Validación probabilidades

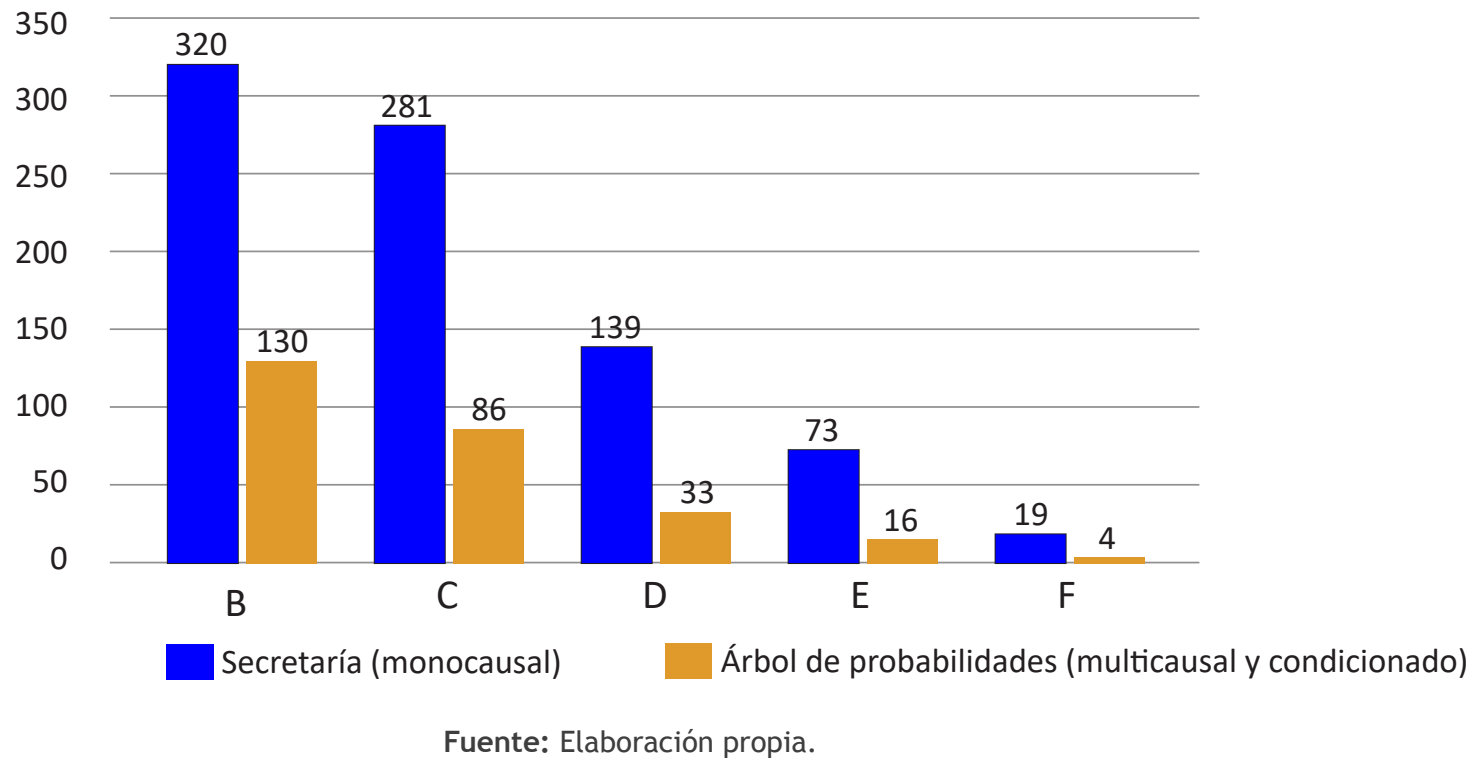


Al pensar en la correlación entre variables, la probabilidad de accidentes disminuye, debido a que se no está enfocando en una sola variable como lo hace la Secretaría Distrital de Movilidad. Al ampliar la cantidad de variables en el evento de que ocurra un accidente conllevará a que sea menos probable que este se materialice.

Sobre la discusión de los resultados obtenidos se tiene lo siguiente:

1. Cabe anotar que las probabilidades frecuenciales se basaron en los datos históricos de registro oficial de accidentes donde estuvo involucrada una bicicleta, se debe observar que esta fuente presenta un subregistro debido a lo oneroso de la denuncia y trámite legal.

2. El uso de probabilidad frecuencial asume que la probabilidad del suceso se estabiliza si se repite el fenómeno infinitas veces. Sin embargo, en la práctica es imposible repetir algo infinitas veces. Algunos autores la definen como la única forma empirica de definir probabilidades.

3. Aunque el árbol de sucesos es una buena representación de la multicausalidad de la accidentalidad, la información del árbol aquí construido debe alimentarse con nueva informacion de otras dimensiones que afectan la accdeintalidad vial: variables en entorno (riesgo de hurto por ejemplo), y variables de la infraestructura, asi como condiciones del uso de la bicicleta.

4. No se encontraron estudios previos sobre esta temática específica, se estudia más los medios motorizados de movilidad.

\section{CONCLUSIONES}

Para promover el uso de la bicicleta como medio de movilidad en Bogotá, no solo se debe incluir los beneficios, sino además un análisis de los factores de riesgo, en este caso de accidentalidad, para así contribuir a la movilidad de manera consiente como se hace con los otros medios de movilidad como el automóvil.

El análisis multicausal de las variables que ocasiona accidentalidad en movilidad en bicicleta evidencia la necesidad de obtener más información sobre los eventos que desencadenan en un accidente. Es decir, la mejora en la investigación de accidentes de tránsito con bicicletas.
Los datos históricos tienen una orientación monocausal y solo analizan variables inherentes al comportamiento del biciusuario, desconociendo el efecto de variables del entorno, del estado la infraestructura, entre otros.

La variable más importante en el árbol de sucesos es B, que significa: no obedecer las señales de tránsito; lo cual implica que se deben fortalecer las campañas y políticas públicas de seguridad vial con énfasis en prevenir los accidentes por esta causa. Esto pasa por procesos de autocuidado del biciusuario y de manejo defensivo en la vía.

Por medio del modelo de probabilidad se determinó que las variables que afectan con mayor influencia el proceso de movilidad en bicicleta son: desobedecer señales, transitar en contravía, adelantar cerrando y no mantener distancia de seguridad [24]. Estas variables son las principales variables a tener en cuenta para plantear estrategias para disminuir la accidentalidad.

\section{RECONOCIMIENTOS}

Gran agradecimiento a la ingeniera Sonia Meneses y a todo el personal de la Universidad Libre que colaboraron y contribuyeron para que este proyecto culminara; al igual que a la familia de cada uno de nosotros quienes fueron los pilares y fuerza para poder concluir con este proyecto.

\section{FINANCIAMIENTO}

Proyecto de investigación financiado por la Universidad Libre y recursos propios de los investigadores.

\section{DISPONIBILIDAD DE DATOS Y MATERIALES}

Secretaría de Movilidad: tel: (031) 3649400

Correo: judicial@movilidadbogota.gov.co

Universidad Libre: tel: 018000180560

Correo: ingeniería_industrial@unilibrebog.edu.co

Deison Duarte: tel: 3176358774

Correo: deisons.duartev@unilibrebog.edu.co

Katherin Lopez: tel: 3192650421

Correo: katherinp.lopezr@unilibrebog.edu.co

Sonia Meneses: tel: 3003773522

Correo:sonial.menesesv@unilibre.edu.co 


\section{CONTRIBUCIÓN DE AUTORES}

Deison Duarte: investigador 1, contribuyó en los antecedentes y validación del modelo de probabilidad.

Katherin López: investigadora 2, contribuyó en la metodología y el modelo de probabilidad.

Sonia Meneses: directora del semillero de investigación, diseñó, estructuró e hizo seguimiento de la investigación y las herramientas a usar.

\section{APROBACIÓN ÉTICA Y CONSENTIMIENTO DE LOS PARTICIPANTES}

Con base en la resolución $n .^{\circ} 1$ de agosto 10 del 2015, emitido por la Conciliatura de la Universidad Libre, por el cual se estructura el Comité de Ética y Bioética de la Investigación, el proyecto de investigación del cual es parte este artículo cumple con lo establecido en el Artículo 6 sobre los aspectos a considerar.

\section{CONFLICTO DE INTERESES}

Sonia Meneses Velosa, con cédula de ciudadanía n. ${ }^{\circ}$ 39.544.879, Deison Stevens Duarte Vargas, identificado con cédula de ciudadanía $n .^{\circ}$ 1.016.094.230 de Bogotá, y Katherin Paola López Rodríguez identificada con cédula de ciudadanía n. ${ }^{\circ} 1.014 .266 .526$ informamos que el presente proyecto está libre de conflicto de intereses.

\section{REFERENCIAS}

[1] R. Flores, R. Alberto, T. A. Pablo, P. C. Felipe y L. Natalia, Ciclo-inclusión en América Latina y el Caribe: Guía para impulsar el uso de la bicicleta, Banco Interamericano de Desarrollo, feb., 2015. [En línea].Disponible en: https:// publications.iadb.org/es/publicacion/13841/ ciclo-inclusion-en-america-latina-y-elcaribe-guia-para-impulsar-el-uso-de-la.

[2] A. M. Cuevas, "Más de 835.000 personas se mueven en bicicleta en Bogotá", Bogotá: Alcaldia Mayor de Bogotá, jun. 28, 2018. [En línea]. Disponible en: http://www. bogota.gov. $\mathrm{co} /$ temas-de-ciudad/movilidad/cuantas-personas-se-mueven-en-bicicleta-en-bogota. [Último acceso: 10 Octubre 2018].
[3] Secretaria Distrital de Movilidad, "La bicicleta en Bogotá (Plan Bici)”, Alcaldia Mayor de Bogotá, 2016. [En línea]. Disponible en: https: / /www.movilidadbogota.gov.co/ web/plan_bici [último acceso: 10 de junio de 2018].

[4] D. Duarte, K. López, S. Meneses, "Caracterización de riesgos en la accidentalidad de biciusuarios. Bogotá - Engativá", Revista de Ingeniería Industrial Actualidad y Nuevas Tendencias, vol. 6, n. ${ }^{\circ} 21$, pp. p. 93-108, 2018.

[5] F. Motoa y J. Cerón, "La apuesta del POT: menos carros, pero más transporte público y ciclas", El Tiempo, 15 dic. 2018. [En línea]. Disponible en: https://www.eltiempo.com/ bogota/propuestas-para-el-pot-menos-carros-y-mas-transporte-publico-en-bogota-305626. [Último acceso: 10 de junio de 2018].

[6] R. Ríos-Flores, A. P. Taddia, C. F. Pardo y N. Lleras, Ciclo-inclusión en América Latina y el Caribe, México: Banco Interamericano de Desarrollo, 2015.

[7] J. Jiménez-Castro, T. Díaz-Anacona y S. Meneses-Velosa, "Caracterización de los riesgos de movilidad en bicicleta en una institución de educación superior en la localidad de Engativá, Bogotá", Avances. Investigación en Ingeniería, vol. 14, n. ${ }^{\circ}$ 1, pp. 46-61, 2017.

[8] LogMovilidad, Formulación del plan maestro de movilidad para Bogotá D. C., que incluye ordenamiento de estacionamientos, Bogotá D. C., 2014.

[9] M. Giraldo, “Más de 2 mil ciclistas han creado su usuario y 475 ya cuentan con el sticker de marcación en sus bicicletas", Bogotá, 3 dic. 2018. [En línea]. Disponible en: http://www. bogota.gov.co/temas-de-ciudad/movilidad/ como-registrar-la-bicicleta-en-bogota [último acceso: 12 de junio de 2018].

[10] Alcaldía Mayor de Bogotá, Anuario de Siniestralidad Vial de Bogotá, Bogotá D. C., 2017.

[11] Secretaria Distrital de Movilidad, "Siniestros ciclistas Engativá”, Bogotá D. C., 2018.

[12] L. E. Beltrán-Rueda, "El decálogo para ser un buen biciusuario, con o sin motor", 8 abr. 2016. [En línea]. Disponible en: http:// www.eltiempo.com/archivo/documento/ CMS-16558651. 
[13] R. Marqués, V. Hernández-Herradora, M. Calvo-Salazar y J.A.García-Cebrián, "How infrastructure can promote cycling in cities: Lessons from Seville", Research in Transportation Economics, vol. 53, n 1, pp. 31-44, 2015.

[14] R. Aldred, “Incompetent or Too Competent? Negotiating Everyday Cycling Identities in a Motor Dominated Society", Mobilities, vol. 8, n. ${ }^{\circ} 2$ DOI: https: //doi.org/10.1080/17450101 .2012.696342, pp. 252-271, 2012.

[15] H. Ferrando, M. Martín y J. López, « estudio sobre las estrategias de promoción de la bicicleta como medio de transporte en las ciudades españolas", jul. 2011. [En línea]. Disponible en: http://www.dgt.es/Galerias/ seguridad-vial/investigacion/estudios-e-informes/INFORME-ESTUDIO-SOBRE-LAS-ESTRATEGIAS-DE-PROMOCION-DE-LA-BICICLETA-9. pdf [último acceso: 11 Junio 2018].

[16] J.-P. Wolff, "Infrastructures de transports et conflits", Toulouse: Presses Universitaires du Midi, 2015. [En línea]. Disponible en: https:// journals.openedition.org/soe/2119 [último acceso: 19 de agosto de 2018].

[17] T. L. V. Torrado y I. H. Larrauri, "La bicicleta como medio de transporte. Puntos de vista de las personas usuarias y expertas", Vitoria-Gasteiz: Servicio Central de Publicaciones del Gobierno Vasco, 2006. [En línea]. Disponible en: http://www.bizkaia. eus / fitxategiak/07/Mediateka / 2_La\%20 bicicleta\%20como\%20medio\%20de\%20transporte_cas.pdf?hash=b3bb03617e41ec3c8e0f8aa09234b263 [último acceso: 9 de agosto de 2018].
[18] Ministerio de Trabajo y Asuntos Sociales España, "NTP 328: Análisis de riesgos mediante el árbol de sucesos", 1994. [En línea]. Disponible en: https://www.insst. es/documents/94886/326827/ntp_328. pdf/278a99c2-0f07-461f-8292-18ae44974d12

[19] Cámara de Comercio de Bogotá y Universidad de los Andes, "Observatorio de Movilidad - Reporte Anual de Movilidad 2015", 12 sept. 2015. [En línea]. Disponible en: https://bibliotecadigital.ccb.org.co/handle/11520/18119[último acceso: 15 de septiembre de 2018].

[20] N Dang, et al "Un enfoque basado en el árbol de eventos dinámicos para el modelado de secuencia de accidentes probabilísticos: dinámica y variabilidades en el medio", Ingeniería de confiabilidad y seguridad del sistema, vol. 142, $\mathrm{n}^{\circ}$. 1, pp. 78-91, 2015. En línea: https://www. sciencedirect.com/ science/article/abs/pii/ S0951832015001234

[21] A. Raiyan, S. Das y M. Islam, "Event tree analysis of marine accidents in Bangladesh", Procedia Engineering, vol. 194, $\mathrm{n}^{\circ}$. 1, pp. 276-283, 2017.

[22] P. V. E. Duarte, "Departamento de Ciencias Matemáticas. Iniciación al Cálculo”, Universidad Eafit, 2019.https://repository.eafit.edu. co/handle/10784/9550

[23] L. N. Ramírez Castañeda, “Caracterización de riesgos en el eslabón de almacenamiento y distribución en una central de abastecimiento agrícola", Gestión de las operaciones en las cadenas de suministro de alimentos, L. N. Ramírez Castañeda, comp. Bogotá, D. C, Universidad Libre, 2018. 